\title{
HIGH-FREQUENCY AVERAGING IN SEMI-CLASSICAL HARTREE-TYPE EQUATIONS
}

\author{
JOHANNES GIANNOULIS, ALEXANDER MIELKE, AND CHRISTOF SPARBER
}

\begin{abstract}
We investigate the asymptotic behavior of solutions to semi-classical Schrödinger equations with nonlinearities of Hartree type. For a weakly nonlinear scaling, we show the validity of an asymptotic superposition principle for slowly modulated highly oscillatory pulses. The result is based on a highfrequency averaging effect due to the nonlocal nature of the Hartree potential, which inhibits the creation of new resonant waves. In the proof we make use of the framework of Wiener algebras.
\end{abstract}

\section{INTRODUCTION AND MAIN RESULT}

In this work we are interested in the asymptotic behavior for $0<\varepsilon \ll 1$ of the following nonlinear Schrödinger equation

$$
i \varepsilon \partial_{t} \mathrm{u}^{\varepsilon}=-\frac{\varepsilon^{2}}{2} \Delta \mathrm{u}^{\varepsilon}+\left(K *\left|\mathrm{u}^{\varepsilon}\right|^{2}\right) \mathrm{u}^{\varepsilon},\left.\quad \mathrm{u}^{\varepsilon}\right|_{t=0}=\mathrm{u}_{0}^{\varepsilon},
$$

where $(t, x) \in \mathbb{R}_{+} \times \mathbb{R}^{d}, d \in \mathbb{N}$. This model describes the time-evolution of a complex-valued field $u^{\varepsilon}(t, x)$, under the influence of a Hartree-type nonlinearity (cf. [17] for a broader introduction) Above, $K=K(x)$ denotes a given real-valued interaction kernel, to be specified in detail below, and "*" denotes the convolution w.r.t. $x \in \mathbb{R}^{d}$. The scaling of (1.1) for small $\varepsilon>0$ corresponds to the semiclassical regime, i.e. the regime of high-frequency solutions $\mathrm{u}^{\varepsilon}(t, x)$, which can be approximated via geometric optics.

The asymptotic behavior of (1.1) as $\varepsilon \rightarrow 0_{+}$has been studied by several authors, mainly for the case $K(x)= \pm \frac{1}{|x|}$ : For example in [14,16,18], Wigner measure techniques are invoked, which however require mixed states and thus can not be applied to our situation. In the one-dimensional case, this constraint can be overcome [19], but uniqueness of the limiting Wigner measure for $t>0$ is still open. Turning to multi-scale WKB expansions, which are typically valid for short times only, a variety of asymptotic results can be found in $[1,7,10,15]$, provided that $u_{0}^{\varepsilon}$ is given as a single-phase WKB initial data, i.e

$$
\mathrm{u}_{0}^{\varepsilon}(x)=a^{\varepsilon}(x) e^{i \varphi(x) / \varepsilon},
$$

with given ( $\varepsilon$-independent) phase $\varphi(x) \in \mathbb{C}$ and amplitude $a^{\varepsilon}(x) \in \mathbb{R}$, such that, asymptotically, $a^{\varepsilon} \sim a_{0}+\varepsilon a_{1}+\varepsilon^{2} a_{2}+\ldots$.

2000 Mathematics Subject Classification. 35B40, 35C20, 81Q20.

Key words and phrases. Nonlinear Schrödinger equation, Hartree nonlinearity, high-frequency asymptotics, WKB approximation.

C.S. has been supported by the Royal Society via his University Research Fellowship. A.M. was partially supported by DFG via project D14 of MATHEON. 
In the present work we are interested in generalizing these studies to the case of (a superposition of) several WKB waves. Due to the expected nonlinear interaction between high-frequency waves (i.e. the appearance of resonances), this problem is notoriously difficult, even on a formal level. We shall therefore simplify the situation considerably by turning our attention to the case of (asymptotically) small initial data corresponding to modulated plane-waves. More precisely, we consider $u_{0}^{\varepsilon}(x)=$ $\varepsilon^{\alpha / 2} u_{0}^{\varepsilon}(x)$, where $\alpha \geqslant 1$ and $u_{0}^{\varepsilon}(x)$ is given by a superposition of $\varepsilon$-oscillatory planewaves, i.e.

$$
u_{0}^{\varepsilon}(x)=\sum_{j=1}^{J} a_{j}(x) e^{i k_{j} \cdot x / \varepsilon}, \quad k_{j} \in \mathbb{R}^{d}
$$

with amplitudes $a_{j}(x) \in \mathbb{C}, j \in\{1, \ldots, J\} \subseteq \mathbb{N}$. Here, and in the following, we shall assume for the sake of simplicity that the initial amplitudes $a_{j}$ do not depend on $\varepsilon$, since we shall only be interested in the leading order asymptotics. A generalization to amplitudes admitting an asymptotic expansion in $\varepsilon$ is then straightforward. Without restriction of generality we shall also assume that

$$
k_{j} \neq k_{\ell} \text { for all } j \neq \ell \in \Gamma:=\{1, \ldots, J\} \subseteq \mathbb{N},
$$

where $J \in \mathbb{N} \cup\{\infty\}$ and $\{1, \ldots, \infty\}$ means simply $\mathbb{N}$.

By rescaling $u^{\varepsilon}=\varepsilon^{-\alpha / 2} \mathrm{u}^{\varepsilon}$, we can rewrite the considered model into

$$
i \varepsilon \partial_{t} u^{\varepsilon}=-\frac{\varepsilon^{2}}{2} \Delta u^{\varepsilon}+\varepsilon^{\alpha}\left(K *\left|u^{\varepsilon}\right|^{2}\right) u^{\varepsilon},\left.\quad u^{\varepsilon}\right|_{t=0}=u_{0}^{\varepsilon},
$$

where the initial data is now of order one but the equation displays an (asymptotically) small nonlinearity. We shall from now on take on this point of view since it looks more natural from the physical point of view. In addition, it is well known (see e.g. [3, 4]) that the choice $\alpha=1$ for $(1.3)$ is critical as far as semiclassical behavior is concerned (see Section 2 for more details). We shall therefore pay particular attention to this case. We remark that the same asymptotic scaling has been used in [5, 6] for Schrödinger equations with (gauge invariant) power-law nonlinearities $\propto|u|^{2 \sigma} u, \sigma \in \mathbb{N}$. In particular, in [5] the problem of high-frequency wave interaction is exhaustively studied in the case $\sigma=1$ for which a geometric description of all possible nonlinear resonances is given.

However, for the considered case of Hartree type nonlinearities, the situation is very different, due to the nonlocal nature of $K *\left|u^{\varepsilon}\right|^{2}$. Since we expect the solution $u^{\varepsilon}(t, x)$ to be given asymptotically as a superposition of highly oscillatory waves, we clearly can not regard the Hartree term to be slowly varying (as in the case of a single wave). The notion of a resonance though, seems to be not clearly defined in this case. A more sophisticated analysis of the oscillatory structure of $K *\left|u^{\varepsilon}\right|^{2}$ is needed therefore. As we shall see, the nonlocal nature of the Hartree potential yields a kind of averaging effect. In particular no new highly oscillatory phases are created in leading order (via resonances), in sharp contrast to the situation for local nonlinearities.

In order to be more precise, we need to introduce some notation: The Fourier transform of $f \in L^{1} \cap L^{2}\left(\mathbb{R}^{d}\right)$ will be denoted as

$$
(\mathcal{F} f)(\xi) \equiv \widehat{f}(\xi)=\frac{1}{(2 \pi)^{d / 2}} \int_{\mathbb{R}^{d}} f(x) e^{-i x \cdot \xi} d x .
$$


Our analytical approach will be based on the use of the Wiener Algebra (see Section 3 for more details). Within this framework it turns out that the natural space for the amplitudes $a=\left(a_{j}\right)_{j \in \Gamma}$ is given as follows.

\section{Definition 1.1.}

$$
\mathcal{A}\left(\mathbb{R}^{d}\right):=\left\{a=\left(a_{j}\right)_{j \in \Gamma}:\left(\widehat{a}_{j}\right)_{j \in \Gamma} \in \ell^{1}\left(\Gamma ; L^{1}\left(\mathbb{R}^{d}\right)\right)\right\},
$$

$\Gamma=\{1, \ldots, J\} \subseteq \mathbb{N}$, equipped with the norm

$$
\|a\|_{\mathcal{A}\left(\mathbb{R}^{d}\right)}=\sum_{j=1}^{J}\left\|\widehat{a}_{j}\right\|_{L^{1}\left(\mathbb{R}^{d}\right)} .
$$

We are now in the position to state the main theorem of this work.

Theorem 1.2. For $d \geqslant 1$, consider the Cauchy problem (1.3) with $\alpha \geqslant 1$, subject to initial data $u_{0}^{\varepsilon}$ of the form (1.2), where the initial amplitudes $a_{j} \in L^{2}\left(\mathbb{R}^{d}\right) \cap \mathcal{A}\left(\mathbb{R}^{d}\right)$ are such that $\left(\partial_{x}^{p} a_{j}\right)_{j \in \Gamma} \in \mathcal{A}\left(\mathbb{R}^{d}\right)$ for all $|p| \leqslant 2$. In addition assume

$$
|\Lambda|_{\infty}^{-1}:=\inf \left\{\left|k_{\ell}-k_{m}\right|: \ell, m \in \Gamma, \ell \neq m\right\}>0,
$$

and let the interaction kernel $K$ satisfy $(1+|\xi|) \widehat{K}(\xi) \in L^{\infty}\left(\mathbb{R}^{d}\right)$.

Then, for all $T>0$ there exists $C, \varepsilon_{0}>0$, such that for any $\varepsilon \in\left(0, \varepsilon_{0}\right)$, the exact solution to (1.3) can be approximated by

$$
\left\|u^{\varepsilon}-u_{\text {app }}^{\varepsilon}\right\|_{L^{\infty}\left([0, T] \times \mathbb{R}^{d}\right)} \leqslant C \varepsilon^{\beta} \quad \text { with } \beta= \begin{cases}1 & \text { if } \alpha \in\{1\} \cup[2, \infty), \\ \alpha-1 & \text { if } \alpha \in(1,2) .\end{cases}
$$

Here the approximate solution $u_{\mathrm{app}}^{\varepsilon}$ is given by

$$
u_{\text {app }}^{\varepsilon}(t, x)=\sum_{j=1}^{J} a_{j}\left(x-t k_{j}\right) e^{i S_{j}(t, x)} e^{i k_{j} \cdot x / \varepsilon-i t\left|k_{j}\right|^{2} /(2 \varepsilon)},
$$

where $S_{j}(t, x) \in \mathbb{R}$ is defined in (2.10) if $\alpha=1$ and $S_{j}(t, x)=0$ if $\alpha>1$, respectively.

In (1.6), the total number of (highly oscillatory) phases $J \in \mathbb{N} \cup \infty$, is the same as for the initial data (1.2). Hence, no new phases are created by the nonlinearity. Nonlinear effects only show up in leading order via self-modulation of the amplitudes (provided $\alpha=1$ ). The condition (1.4) can be seen as a small divisor assumption required in the case of infinitely many phases, though, not of the same type as the one used in [5]. Obviously, (1.4) is always satisfied if $J<+\infty$.

Note that if $\alpha \in \mathbb{N}$, the error of our approximation is at least of the order $\mathcal{O}(\varepsilon)$ and thus smaller than the size of the original (small amplitude) solution. However, we see from (1.5) that if $\alpha \in(1,2)$ the error becomes worse as $\alpha \rightarrow 1_{+}$. This problem can in principle be overcome if one allows the leading order amplitudes to be mildly dependent on $\varepsilon$ themselves, cf. Remark 2.2 below.

Finally, one should note that under the general assumptions of this work, we can not infer global well posedness of equation (1.3) in the Wiener space. The usual arguments for proving global existence (see e.g. [17]) involve the conservation of the $L^{2}$ norm, which also holds in our case. However, this is not sufficient to control the nonlinearity $\left(K *|u|^{2}\right) u$ in the Wiener space. Nevertheless, the above theorem shows that for initial data $u_{0}^{\varepsilon}$ in the form (1.2), the solutions cannot blow up too fast: If $T^{\varepsilon}>0$ denotes the blow-up time, then $T^{\varepsilon} \rightarrow+\infty$ as $\varepsilon \rightarrow 0_{+}$. 
Remark 1.3. A particular example for $K$, satisfying the assumptions is given by the one considered in [2, equation (15)] and more importantly by the so-called Yukawa potential

$$
K(x)= \pm \frac{e^{-\lambda|x|}}{|x|}, \quad x \in \mathbb{R}^{3}, \lambda>0,
$$

for which the corresponding Fourier transform is found to be

$$
\widehat{K}(\xi)= \pm \frac{1}{\lambda^{2}+|\xi|^{2}}
$$

Clearly, $(1+|\xi|) \widehat{K} \in L^{\infty}\left(\mathbb{R}^{3}\right)$ in this case. Note that in the limit $\lambda \rightarrow 0$ we obtain the Fourier transform of the Coulomb potential $K(x)= \pm \frac{1}{|x|}$, which, however, is too singular, to directly apply our theorem. It remains an interesting open problem to establish the same result for the Coulomb case in $d=3$.

The paper is organized as follows: In Section 2, we formally derive the approximate solution and draw some further conclusions from it. In Section 3 we set up the Wiener framework for the exact and the approximate solution. Finally, we prove the required estimates on the remainder of the approximation and consequently state the proof of Theorem 1.2 in Section 4.

\section{Derivation of the approximate solution}

We seek an approximation of solutions to (1.3) in the form

$$
u_{\mathrm{app}}^{\varepsilon}(t, x)=\sum_{j=1}^{J} A_{j}(t, x) e^{i \phi_{j}(t, x) / \varepsilon} .
$$

Assuming for the moment sufficient smoothness for $A_{j}$ and $\phi_{j}$, we can plug this ansatz into (1.3), which yields

$$
i \varepsilon \partial_{t} u_{\mathrm{app}}^{\varepsilon}+\frac{\varepsilon^{2}}{2} \Delta u_{\mathrm{app}}^{\varepsilon}-\varepsilon^{\alpha}\left(K *\left|u_{\mathrm{app}}^{\varepsilon}\right|^{2}\right) u_{\mathrm{app}}^{\varepsilon}=\sum_{n=0}^{2} \varepsilon^{n} X_{n}+\varepsilon^{\alpha}\left(Y+Y_{R}\right),
$$

where we denote

$$
\begin{aligned}
& X_{0}=-\sum_{j=1}^{J} e^{i \phi_{j} / \varepsilon}\left(\partial_{t} \phi_{j}+\frac{1}{2}\left|\nabla \phi_{j}\right|^{2}\right) A_{j}, \\
& X_{1}=i \sum_{j=1}^{J} e^{i \phi_{j} / \varepsilon}\left(\partial_{t} A_{j}+\nabla A_{j} \cdot \nabla \phi_{j}+\frac{1}{2} A_{j} \Delta \phi_{j}\right),
\end{aligned}
$$

and also

$$
X_{2}=\frac{1}{2} \sum_{j=1}^{J} e^{i \phi_{j} / \varepsilon} \Delta A_{j}
$$


These terms are the same as in the linear case $K \equiv 0$. Due to the presence of the Hartree type nonlinearity, we also obtain

$$
\begin{aligned}
Y & =-\sum_{j=1}^{J} e^{i \phi_{j} / \varepsilon}\left(K * \sum_{\ell=1}^{J}\left|A_{\ell}\right|^{2}\right) A_{j} \\
Y_{R} & =-\sum_{j=1}^{J} e^{i \phi_{j} / \varepsilon}\left(K * \sum_{\substack{\ell, m=1 \\
\ell \neq m}}^{J}\left(A_{\ell} \bar{A}_{m} e^{i\left(\phi_{\ell}-\phi_{m}\right) / \varepsilon}\right)\right) A_{j} .
\end{aligned}
$$

Obviously, $Y_{R}$ carries high-frequency oscillations, which are not captured by our ansatz (2.1). Thus we need to develop a more careful analysis in the following, which shows that $Y_{R}$ is of higher order.

Ignoring this problem for the moment, we consequently aim to eliminate equal powers of $\varepsilon$. Hence, in leading order, we set $X_{0}=0$, which is equivalent to the Hamilton-Jacobi equation

$$
\partial_{t} \phi_{j}+\frac{1}{2}\left|\nabla \phi_{j}\right|^{2}=0,\left.\quad \phi_{j}\right|_{t=0}=k_{j} \cdot x .
$$

Solutions to (2.6) determine the characteristic high-frequency oscillations present in $u_{\mathrm{app}}^{\varepsilon}$. In our case, they are easily found to be

$$
\phi_{j}(t, x)=k_{j} \cdot x-\frac{t}{2}\left|k_{j}\right|^{2} .
$$

These phases obviously solve $(2.6)$ for all $(t, x) \in \mathbb{R} \times \mathbb{R}^{d}$, i.e. no caustics appear in our study.

In the next step we set $X_{1}=0$ if $\alpha>1$ and $X_{1}+Y=0$ if $\alpha=1$ (note that we do not include $Y_{R}$ here). Comparing the prefactors of the terms multiplied by $e^{i \phi_{j} / \varepsilon}$, yields the following system of transport equations for the amplitudes:

$$
\partial_{t} A_{j}+k_{j} \cdot \nabla A_{j}= \begin{cases}0 & \text { if } \alpha>1, \\ -i V_{\mathrm{eff}}(A) A_{j} & \text { if } \alpha=1,\end{cases}
$$

where we have used the fact that $\Delta \phi_{j} \equiv 0$, in view of (2.7). For $\alpha=1$, the effective (nonlinear) potential $V_{\text {eff }}(A)$ is given by

$$
V_{\text {eff }}(A):=K *\left(\sum_{\ell=1}^{J}\left|A_{\ell}\right|^{2}\right) .
$$

We see that for $\alpha>1$ no nonlinear effects are present in transport equations for the leading order amplitudes. The case $\alpha=1$ is therefore seen to be critical as far as semi-classical asymptotics is concerned.

Lemma 2.1. The transport equation (2.8) with initial data $\left(a_{j}\right)_{j \in \Gamma} \in L^{2} \cap \mathcal{A}\left(\mathbb{R}^{d}\right)$ admits global-in-time solutions $A \in C\left([0, \infty) ; L^{2} \cap \mathcal{A}\left(\mathbb{R}^{d}\right)\right)$, which can be written in the form

$$
A_{j}(t, x)=a_{j}\left(x-t k_{j}\right) e^{i S_{j}(t, x)}
$$

where $S_{j} \equiv 0$ for $\alpha>1$ and $S_{j} \in C\left([0, \infty) \times \mathbb{R}^{d}\right)$ for $\alpha=1$ is given by

$$
S_{j}(t, \cdot)=-\int_{0}^{t}\left(K * \sum_{\ell=1}^{J}\left|a_{\ell}\left(\cdot+(\tau-t) k_{j}-\tau k_{\ell}\right)\right|^{2}\right) d \tau \text {. }
$$


In particular we have mass conservation for each individual mode

$$
\left\|A_{j}(t, \cdot)\right\|_{L^{2}\left(\mathbb{R}^{d}\right)}=\left\|a_{j}\right\|_{L^{2}\left(\mathbb{R}^{d}\right)}, \quad \forall t \in \mathbb{R} .
$$

In contrast to $\phi_{j}$ the phases $S_{j}$ are only slowly varying, i.e. they oscillate with frequencies larger than $1 / \varepsilon$. They describe the nonlinear self-modulation of the amplitudes but do not show up in quadratic quantities, like the mass density $\left|A_{j}\right|^{2}$ etc.

Proof. By multiplying (2.8) with $\bar{A}_{j}$ and taking the real part, we see

$$
\left(\partial_{t}+k_{j} \cdot \nabla\right)\left|A_{j}\right|^{2}=0,
$$

which yields $\left|A_{j}(t, x)\right|^{2}=\left|a_{j}\left(x-t k_{j}\right)\right|^{2}$ and thus (2.9) and (2.11). Finally, inserting (2.9) into (2.8) and integrating along characteristics, we obtain the expression (2.10) for $S_{j}$.

Having obtained the characteristic phases $\phi_{j}$ and the leading order amplitudes $A_{j}$ we shall now turn our attention towards the remainder, i.e.

$$
R\left(u_{\mathrm{app}}^{\varepsilon}\right)= \begin{cases}\varepsilon^{2} X_{2}+\varepsilon Y_{R} & \text { if } \alpha=1, \\ \varepsilon^{2} X_{2}+\varepsilon^{\alpha}\left(Y+Y_{R}\right) & \text { if } \alpha>1 .\end{cases}
$$

For $\alpha=1$ the term $Y_{R}$ appearing within $R\left(u_{\text {app }}^{\varepsilon}\right)$ is formally of order $\mathcal{O}(\varepsilon)$. Thus, at first glance, $Y_{R}$ seems to be too large to be considered a part of the remainder. It will be our main task to show that $Y_{R}$ is indeed sufficiently small as $\varepsilon \rightarrow 0_{+}$. To this end, we shall rely on the framework of Wiener algebras.

Remark 2.2. In the case $\alpha>1$, there is a some freedom in deriving the system of amplitude equations. Indeed, one could regard $\varepsilon^{\alpha-1}$ merely as a prefactor for the interaction kernel $K$ and solve in the next step $X_{1}+\varepsilon^{\alpha-1} Y=0$. Instead of (2.8), we would consequently get

$$
\partial_{t} A_{j}^{\varepsilon}+k_{j} \cdot \nabla A_{j}^{\varepsilon}=-i \varepsilon^{\alpha-1} V_{\mathrm{eff}}\left(A^{\varepsilon}\right) A_{j}^{\varepsilon} \quad \text { for } \alpha \geqslant 1,
$$

which yields

$$
A_{j}^{\varepsilon}(t, x)=a_{j}\left(x-t k_{j}\right) e^{i \varepsilon^{\alpha-1} S_{j}(t, x)},
$$

with $S_{j}$ given as before. In other words, the leading order amplitudes would become $\varepsilon$-dependent and instead of (2.12) the remainder would have the form

$$
R\left(u_{\mathrm{app}}^{\varepsilon}\right)=\varepsilon^{2} X_{2}+\varepsilon^{\alpha} Y_{R} \text { for } \alpha \geqslant 1 .
$$

We expect that the subsequent analysis can be adapted to the case of $\varepsilon$-dependent amplitudes given by (2.13), and in fact we expect a slightly better error estimate in this case. Namely, we expect (1.5) to hold with $\beta=1$ for all $\alpha \geqslant 1$. However, we hesitate to carry through this approach in full detail, since the separation of slow- and fast scales within the leading order approximation becomes much less apparent. In addition, one usually does not regard (2.13) as a proper leading order amplitude in semi-classical analysis (due to its $\varepsilon$-dependence). We shall therefore resume our analysis with $\varepsilon$-independent $A_{j}$ as in our original ansatz (2.1). 


\section{The WiEnER ALGEBRA FRAMEWORK}

We now present the analytical framework of Wiener algebras which already proved its use in similar circumstances, cf. [5, 8, 12]. We start with the following definition.

Definition 3.1 (Wiener Algebra). We define

$$
W\left(\mathbb{R}^{d}\right):=\left\{f \in \mathcal{S}^{\prime}\left(\mathbb{R}^{d} ; \mathbb{C}\right), \quad\|f\|_{W}:=\|\widehat{f}\|_{L^{1}\left(\mathbb{R}^{d}\right)}<\infty\right\} .
$$

The following properties of $W\left(\mathbb{R}^{d}\right)$ have been proved in $[8,12]$.

\section{Lemma 3.2.}

i. $W\left(\mathbb{R}^{d}\right)$ is a Banach space, continuously embedded into $L^{\infty}\left(\mathbb{R}^{d}\right)$.

ii. $W\left(\mathbb{R}^{d}\right)$ is an algebra, in the sense that the mapping $(f, g) \mapsto f g$ is continuous from $W\left(\mathbb{R}^{d}\right)^{2}$ to $W\left(\mathbb{R}^{d}\right)$, and moreover

$$
\forall f, g \in W\left(\mathbb{R}^{d}\right), \quad\|f g\|_{W} \leqslant\|f\|_{W}\|g\|_{W} .
$$

iii. Let $U^{\varepsilon}(t)=e^{i \varepsilon \frac{t}{2} \Delta}$ denote the free Schrödinger group. Then, for all $t \in \mathbb{R}$, $U^{\varepsilon}(t)$ is unitary on $W\left(\mathbb{R}^{d}\right)$.

Assertion iii. follows from the fact that $\widehat{U}^{\varepsilon}(t)=e^{i t|\xi|^{2} / 2}$, acting as a multiplication operator in Fourier space. From now on, we shall consider the Cauchy problem (1.3) to be posed in $W\left(\mathbb{R}^{d}\right)$. To this end we need the following well-posedness result (which is an adaptation of the one given in [5] to the case of Hartree nonlinearities).

Lemma 3.3. Consider the initial value problem

$$
i \varepsilon \partial_{t} u^{\varepsilon}+\frac{\varepsilon^{2}}{2} \Delta u^{\varepsilon}=\varepsilon^{\alpha}\left(K *\left|u^{\varepsilon}\right|^{2}\right) u^{\varepsilon},\left.\quad u^{\varepsilon}\right|_{t=0}=u_{0}^{\varepsilon}
$$

with $\alpha \geqslant 1$. If $\widehat{K} \in L^{\infty}\left(\mathbb{R}^{d}\right)$ and $u_{0}^{\varepsilon} \in W\left(\mathbb{R}^{d}\right)$ with $\left\|u_{0}^{\varepsilon}\right\|_{W} \leqslant D_{0}$, then there exists a $T_{0}>0$, which depends on $D_{0}$ but not on $\varepsilon$, and a unique solution $u^{\varepsilon} \in$ $C\left(\left[0, T_{0}\right] ; W\left(\mathbb{R}^{d}\right)\right)$ of $(1.3)$.

Proof. Duhamel's formulation of (1.3) reads

$$
\left.u^{\varepsilon}(t)=U^{\varepsilon}(t) u_{0}^{\varepsilon}-i \varepsilon^{\alpha-1} \int_{0}^{t} U^{\varepsilon}(t-\tau)\left(\left(K *\left|u^{\varepsilon}\right|^{2}\right) u^{\varepsilon}\right)\right)(\tau) d \tau .
$$

Denote, for fixed $u_{0}^{\varepsilon}$, the right hand side of this formula by $\Phi^{\varepsilon}\left(u^{\varepsilon}\right)(t)$. From Lemma 3.2 iii. we have

$$
\left.\left\|\Phi^{\varepsilon}\left(u^{\varepsilon}\right)(t)\right\|_{W} \leqslant D_{0}+\varepsilon^{\alpha-1} \int_{0}^{t} \|\left(\left(K *\left|u^{\varepsilon}\right|^{2}\right) u^{\varepsilon}\right)\right)(\tau) \|_{W} d \tau
$$

In order to control the nonlinear term, we need to estimate expressions of the form $(K *(u v)) w$ in $W\left(\mathbb{R}^{d}\right)$. To this end, we first use Lemma 3.2 ii, to obtain

$$
\|(K *(u v)) w\|_{W} \leqslant\|K *(u v)\|_{W}\|w\|_{W} .
$$

By Hölder's inequality we also get

$$
\|K *(u v)\|_{W}=\|\widehat{K *(u v)}\|_{L^{1}} \leqslant\|\widehat{K}\|_{L^{\infty}}\|u v\|_{W}
$$

and applying again Lemma 3.2 ii, we arrive at

$$
\|(K *(u v)) w\|_{W} \leqslant\|\widehat{K}\|_{L^{\infty}}\|u\|_{W}\|v\|_{W}\|w\|_{W} \quad \text { for } u, v, w \in W\left(\mathbb{R}^{d}\right) .
$$


Thus, from (3.1) we obtain

$$
\left\|\Phi^{\varepsilon}\left(u^{\varepsilon}\right)(t)\right\|_{W} \leqslant D_{0}+\varepsilon^{\alpha-1}\|\widehat{K}\|_{L^{\infty}} \int_{0}^{t}\left\|u^{\varepsilon}(\tau)\right\|_{W}^{3} d \tau .
$$

Moreover, $u^{\varepsilon} \mapsto \Phi^{\varepsilon}\left(u^{\varepsilon}\right)$ is locally Lipschitz in $U:=C\left([0, T], W\left(\mathbb{R}^{d}\right)\right)$ : If $\left\|u^{\varepsilon}\right\|_{U} \leqslant D$, $\left\|v^{\varepsilon}\right\|_{U} \leqslant D$, then there exists $C=C(D)$ such that

$$
\left\|\Phi^{\varepsilon}\left(u^{\varepsilon}\right)(t)-\Phi^{\varepsilon}\left(v^{\varepsilon}\right)(t)\right\|_{W} \leqslant C(D) \int_{0}^{t}\left\|u^{\varepsilon}(\tau)-v^{\varepsilon}(\tau)\right\|_{W} d \tau, \quad \forall t \in[0, T] .
$$

A fixed point argument in $\left\{u \in U: \sup _{t \in[0, T]}\|u(t)\|_{W} \leqslant D\right\}$, with $D>D_{0}$, for $T=T_{0}$ sufficiently small, then yields Lemma 3.3.

Having set up an existence result for the exact solution $u^{\varepsilon}$ in $W\left(\mathbb{R}^{d}\right)$, we now turn to the approximate solution $u_{\text {app }}^{\varepsilon}$ given by (1.6). To this end, we shall need the following lemma, which shows that the Wiener space is perfectly adapted to our problem.

Lemma 3.4. Let $k_{j} \in \mathbb{R}^{d}, c_{j} \in \mathbb{R}$, and $b \in \ell^{1}\left(\Gamma, W\left(\mathbb{R}^{d}\right)\right)$, and set

$$
f(x, y)=\sum_{j=1}^{J} b_{j}(x) e^{i\left(k_{j} \cdot y+c_{j}\right)} .
$$

Then, for all $\varepsilon>0$ the function $f(\cdot, \cdot / \varepsilon): x \mapsto f(x, x / \varepsilon)$ lies in $W\left(\mathbb{R}^{d}\right)$ with

$$
\|f(\cdot, \cdot / \varepsilon)\|_{W} \leqslant\|b\|_{\mathcal{A}}=\sum_{j \in \Gamma}\left\|b_{j}\right\|_{W} .
$$

Proof. We write

$$
\|f(\cdot, \cdot \varepsilon)\|_{W}=\left\|\sum_{j=1}^{J} e^{i c_{j}} \widehat{b_{j}}\left(\cdot-k_{j} / \varepsilon\right)\right\|_{L^{1}} \leqslant \sum_{j=1}^{J}\left\|\widehat{b_{j}}\left(\cdot-k_{j} / \varepsilon\right)\right\|_{L^{1}}=\sum_{j=1}^{J}\left\|\widehat{b_{j}}\right\|_{L^{1}} .
$$

The last term is, by definition, $\|b\|_{\mathcal{A}}$.

Remark 3.5. This lemma in general does not hold for functions of the form $f(x, y)=\sum_{j=1}^{J} b_{j}(x) e^{i \varphi_{j}(y)}$, with $\varphi_{j}(y) \neq k_{j} \cdot y+c_{j}$. A generalization of our study to non-plane wave WKB phases therefore seems to be a delicate issue (at least within the Wiener framework) and by no means straightforward.

Since the phases $\phi_{j}$ considered in this work are of plane-wave form (2.7), applying Lemma 3.4 with $c_{j}=-\frac{t}{2 \varepsilon}\left|k_{j}\right|^{2}$ to (1.6), we immediately obtain

$$
\left\|u_{\mathrm{app}}^{\varepsilon}(t, \cdot)\right\|_{W} \leqslant\|A(t, \cdot)\|_{\mathcal{A}} .
$$

Similarly, we can estimate the expression (2.3) for $X_{2}$ by

$$
\left\|X_{2}(t, \cdot)\right\|_{W} \leqslant \frac{1}{2}\|\Delta A(t, \cdot)\|_{\mathcal{A}}=\frac{1}{2} \sum_{j=1}^{J}\left\|\Delta A_{j}(t, \cdot)\right\|_{W} .
$$

In addition, we obtain the following estimate for $Y$, defined in (2.4):

$$
\|Y(t, \cdot)\|_{W} \leqslant\left\|K * \sum_{\ell=1}^{J}\left|A_{\ell}(t, \cdot)\right|^{2}\right\|_{W}\|A(t, \cdot)\|_{\mathcal{A}},
$$


where we have used Lemma 3.4 and Lemma 3.2 ii. This can be estimated further similarly to (3.2) by using Lemma 3.4 (with $k_{j}=0, c_{j}=0$ ), as well as Assertion ii. of Lemma 3.2 and the fact that $\ell^{\infty} \subset \ell^{1}$, to obtain

$$
\|Y(t, \cdot)\|_{W} \leqslant\|\widehat{K}\|_{L^{\infty}}\|A(t, \cdot)\|_{\mathcal{A}}^{3} .
$$

In order to close the argument, we consequently require appropriate bounds in $\mathcal{A}\left(\mathbb{R}^{d}\right)$ on the amplitudes $A_{j}(t, \cdot)$, together with their spatial derivatives.

Lemma 3.6. Let $\alpha \geqslant 1$ and $\widehat{K} \in L^{\infty}\left(\mathbb{R}^{d}\right)$. For all $a=\left(a_{j}\right)_{j \in \Gamma} \in \mathcal{A}\left(\mathbb{R}^{d}\right)$, there exists a unique solution $A \in C\left([0, \infty) ; \mathcal{A}\left(\mathbb{R}^{d}\right)\right)$ to the system (2.8). Moreover, if $\left(\partial_{x}^{p} a_{j}\right)_{j \in \Gamma} \in \mathcal{A}\left(\mathbb{R}^{d}\right)$, for $|p| \leqslant 2$, then $\left(\partial_{x}^{p} A_{j}\right)_{j \in \Gamma} \in C\left([0, \infty) ; \mathcal{A}\left(\mathbb{R}^{d}\right)\right)$.

Proof. For $\alpha>1$ the statements of the lemma are immediately clear, since in this case $A_{j}(t, x)=a_{j}\left(x-t k_{j}\right)$, cf. (2.8). For $\alpha=1$ we rewrite (2.8) in its integral form

$$
A_{j}(t, x)=a_{j}\left(x-t k_{j}\right)+\int_{0}^{t} \mathcal{N}(A)_{j}\left(\tau, x+(\tau-t) k_{j}\right) d \tau,
$$

where the nonlinearity $\mathcal{N}(A)_{j}$ is given by

$$
\mathcal{N}(A)_{j}=-i\left(K *\left(\sum_{\ell=1}^{J}\left|A_{\ell}\right|^{2}\right)\right) A_{j}
$$

Invoking the same arguments as for the derivation of (3.6), we obtain

$$
\|\mathcal{N}(A)\|_{\mathcal{A}}=\sum_{j=1}^{J}\left\|\mathcal{N}(A)_{j}\right\|_{W} \leqslant\|\widehat{K}\|_{L^{\infty}}\|A\|_{\mathcal{A}}^{3} .
$$

This shows that $\mathcal{N}(A)$ defines a continuous mapping from $\mathcal{A}^{3}$ to $\mathcal{A}$ and a localin-time existence result immediately follows from the standard Cauchy-Lipschitz theorem for ordinary differential equations. That the solutions $A_{j}$ indeed exist for all $t \geqslant 0$ then follows from the explicit representation (2.9). From the latter we additionally obtain the propagation of regularity, by explicit calculation of $\partial_{x}^{p} A$.

Lemma 3.6 consequently establishes the estimates in $W\left(\mathbb{R}^{d}\right)$ for $u_{\text {app }}^{\varepsilon}, X_{2}$ and $Y$ in a rigorous way. Note however, that the above given estimates do not yield an estimate for the remainder $R\left(u_{\mathrm{app}}^{\varepsilon}\right)$, given by (2.12), since it also includes $Y_{R}$, which we completely ignored so far. We will make up for it in the following section.

\section{Estimates on the Remainder AND PROOF of THE MAin THEOREM}

It remains to estimate in $W\left(\mathbb{R}^{d}\right)$ the term $Y_{R}$ given in (2.5). To this end we shall prove the following key technical result.

Proposition 4.1. Let $Y_{R}$ be defined by (2.5) with plane-wave phases $\phi_{j}$ given by (2.7) and assume $|\Lambda|_{\infty}^{-1}:=\inf \left\{\left|k_{\ell}-k_{m}\right|: \ell, m \in \Gamma, \ell \neq m\right\}>0$. Moreover, let $K$ be such that $\widehat{K} \in L^{\infty}\left(\mathbb{R}^{d}\right)$ and $\widehat{\nabla K} \in L^{\infty}\left(\mathbb{R}^{d}\right)$. Then we have the following bound:

$$
\left\|Y_{R}(t, \cdot)\right\|_{W} \leqslant \varepsilon C_{K}\|A(t, \cdot)\|_{\mathcal{A}}^{2}\left(\|A(t, \cdot)\|_{\mathcal{A}}+\|\nabla A(t, \cdot)\|_{\mathcal{A}}\right),
$$

where $C_{K}>0$ is independent of $\varepsilon$. 
Proof. Recalling the definition of $Y_{R}$ given in (2.5) and taking into account the particular plane-wave form (2.7) of the phases $\phi_{j}$, we obtain from Lemma 3.4 and Assertion ii. of Lemma 3.2 that

$$
\left\|Y_{R}\right\|_{W} \leqslant\|A\|_{\mathcal{A}}\left\|\sum_{\substack{\ell, m=1 \\ \ell \neq m}}^{J} K *\left(A_{\ell} \bar{A}_{m} e^{i\left(\phi_{\ell}-\phi_{m}\right) / \varepsilon}\right)\right\|_{W} .
$$

Using,

$$
e^{i y \cdot k / \varepsilon+z}=-i \varepsilon \frac{k}{|k|^{2}} \cdot \nabla_{y} e^{i y \cdot k / \varepsilon+z} \quad(z \in \mathbb{C}),
$$

we can perform a partial integration w.r.t $y$, and rewrite

$$
\begin{aligned}
& K *\left(A_{\ell} \bar{A}_{m} e^{i\left(\phi_{\ell}-\phi_{m}\right) / \varepsilon}\right)=\int_{\mathbb{R}^{d}} K(x-y) A_{\ell}(y) \bar{A}_{m}(y) e^{i\left(\phi_{\ell}(y)-\phi_{m}(y)\right) / \varepsilon} d y \\
& \quad=i \varepsilon \int_{\mathbb{R}^{d}} \frac{k_{\ell}-k_{m}}{\left|k_{\ell}-k_{m}\right|^{2}} \cdot \nabla_{y}\left(K(x-y) A_{\ell}(y) \bar{A}_{m}(y)\right) e^{i\left(\phi_{\ell}(y)-\phi_{m}(y)\right) / \varepsilon} d y .
\end{aligned}
$$

To show that the boundary terms vanish, assume first that $A_{j} \in \mathcal{S}\left(\mathbb{R}^{d}\right)$, the set of Schwartz functions (for which the boundary terms clearly vanish). Since $\mathcal{S}\left(\mathbb{R}^{d}\right)$ is dense in $L^{1}\left(\mathbb{R}^{d}\right)$, Fourier transformation implies that $\mathcal{S}\left(\mathbb{R}^{d}\right)$ is also dense in $W\left(\mathbb{R}^{d}\right)$. Consequently, the fact that the expressions on both sides are normbounded sesquilinear forms establishes the above formula.

Using $k_{\ell} \neq k_{m} \in \mathbb{R}^{d}$, we know that $\Lambda_{\ell, m}:=\frac{k_{\ell}-k_{m}}{\left|k_{\ell}-k_{m}\right|^{2}} \in \mathbb{R}^{d}$ is well defined and consequently

$$
\begin{aligned}
K *\left(A_{\ell} \bar{A}_{m} e^{i\left(\phi_{\ell}-\phi_{m}\right) / \varepsilon}\right)= & -i \varepsilon\left(\Lambda_{\ell, m} \cdot \nabla K\right) *\left(A_{\ell} \bar{A}_{m} e^{i\left(\phi_{\ell}-\phi_{m}\right) / \varepsilon}\right) \\
& +i \varepsilon K *\left(\left(\Lambda_{\ell, m} \cdot \nabla\left(A_{\ell} \bar{A}_{m}\right)\right) e^{i\left(\phi_{\ell}-\phi_{m}\right) / \varepsilon}\right) .
\end{aligned}
$$

Using the estimate (3.2) and Lemma 3.4 (with $J=1$ ) we get

$$
\begin{aligned}
\left\|K *\left(A_{\ell} \bar{A}_{m} e^{i\left(\phi_{\ell}-\phi_{m}\right) / \varepsilon}\right)\right\|_{W} \leqslant & \varepsilon\left\|\Lambda_{\ell, m} \cdot \widehat{\nabla K}\right\|_{L^{\infty}}\left\|A_{\ell}\right\|_{W}\left\|A_{m}\right\|_{W} \\
& +\varepsilon\|\widehat{K}\|_{L^{\infty}}\left\|\Lambda_{\ell, m} \cdot \nabla\left(A_{\ell} \bar{A}_{m}\right)\right\|_{W} .
\end{aligned}
$$

Invoking again Lemma 3.4 (with $k_{j}=0, c_{j}=0$ ) and Lemma $3.2 \mathrm{ii}$, we conclude

$$
\begin{aligned}
& \left\|\sum_{\substack{\ell, m=1 \\
\ell \neq m}}^{J} K *\left(A_{\ell} \bar{A}_{m} e^{i\left(\phi_{\ell}-\phi_{m}\right) / \varepsilon}\right)\right\|_{W} \\
& \leqslant \varepsilon \sum_{\substack{\ell, m=1 \\
\ell \neq m}}^{J}\left(d\left|\Lambda_{\ell, m}\right|\|\widehat{\nabla K}\|_{L^{\infty}}\left\|A_{\ell}\right\|_{W}\left\|A_{m}\right\|_{W}+2\|\widehat{K}\|_{L^{\infty}}\left|\Lambda_{\ell, m}\right|\left\|\nabla A_{\ell}\right\|_{\mathcal{A}}\left\|A_{m}\right\|_{W}\right) \\
& \leqslant \varepsilon|\Lambda|_{\infty}\|A\|_{\mathcal{A}}\left(d\|\widehat{\nabla K}\|_{L^{\infty}}\|A\|_{\mathcal{A}}+2\|\widehat{K}\|_{L^{\infty}}\|\nabla A\|_{\mathcal{A}}\right)
\end{aligned}
$$

where $\|\widehat{\nabla K}\|_{L^{\infty}}=\max _{n=1, \ldots, d}\left\|\widehat{\partial_{x_{n}} K}\right\|_{L^{\infty}}$, and

$$
\|\nabla A\|_{\mathcal{A}}=\sum_{\ell=1}^{J}\left\|\nabla A_{\ell}\right\|_{\mathcal{A}}=\sum_{\ell=1}^{J} \sum_{n=1}^{d}\left\|\partial_{x_{n}} A_{\ell}\right\|_{W}
$$

This, together with (4.2) yields the estimate (4.1). 
Remark 4.2. Proposition 4.1 shows that $\left\|Y_{R}\right\|_{W}=\mathcal{O}(\varepsilon)$ and thus can indeed be considered a part of the remainder. Note that in the proof it is essential to invoke a stationary-phase type argument first, before starting to take estimates. In fact we would not succeed to show that $\left\|Y_{R}\right\|_{W}=\mathcal{O}(\varepsilon)$, if we would estimate $Y_{R}$ in its original form.

By combining the results of Lemma 3.6 and Proposition 4.1, we obtain the following result.

Corollary 4.3. Under the assumptions of Lemma 3.6 and Proposition 4.1, there exists, for every $T>0$, a constant $C_{R}(T)>0$, independent of $\varepsilon>0$, such that the remainder $R\left(u_{\mathrm{app}}^{\varepsilon}\right)$ given by $(2.12)$ satisfies

$$
\left\|R\left(u_{\text {app }}^{\varepsilon}\right)\right\|_{W} \leqslant \varepsilon^{\gamma} C_{R} \quad \forall t \in[0, T], \forall \varepsilon>0,
$$

where

$$
\gamma= \begin{cases}2 & \text { if } \alpha \in\{1\} \cup[2, \infty), \\ \alpha & \text { if } \alpha \in(1,2) .\end{cases}
$$

Proof. Lemma 3.6 guarantees the existence of the norms $\|A(t, \cdot)\|_{\mathcal{A}},\|\nabla A(t, \cdot)\|_{\mathcal{A}}$, $\|\Delta A(t, \cdot)\|_{\mathcal{A}}<\infty$ for all $t \in[0, \infty)$, which are independent of $\varepsilon>0$ and continuous in $t$. Hence, taking their maximum over $t \in[0, T]$, we obtain (4.3) from (3.5), (3.6), (4.1) and the definition $(2.12)$ of $R\left(u_{\mathrm{app}}^{\varepsilon}\right)$.

With the estimate of Corollary 4.3 on $R\left(u_{\text {app }}^{\varepsilon}\right)$ at hand, we can finally state the proof of our main theorem, which follows the basic ideas established in [13] for justifying the nonlinear Schrödinger equation as a modulation equation for dispersive waves.

Proof of Theorem 1.2. We consider a fixed $T>0$ and introduce the following scaled error $r^{\varepsilon}$ between the original solution $u^{\varepsilon}$ to (1.3) subject to the initial data (1.2) and the approximation (1.6):

$$
\varepsilon^{\beta} r^{\varepsilon}:=u^{\varepsilon}-u_{\mathrm{app}}^{\varepsilon},
$$

with a parameter $\beta>0$ to be specified below. Hence, $r^{\varepsilon}(0)=0$. From (3.4) and Lemma 3.6 we know that there exists a constant $C_{A}>0$, independent of $\varepsilon$, such that $\left\|u_{\text {app }}^{\varepsilon}(t, \cdot)\right\|_{W} \leqslant C_{A}$, for all $t \in[0, T]$. Since $r^{\varepsilon}(0)=0$, it follows $\left\|u_{0}^{\varepsilon}\right\|_{W} \leqslant C_{A}$. Consequently, for any $D>C_{A}$, Lemma 3.3 yields the existence of a unique solution $u^{\varepsilon} \in C\left(\left[0, T_{0}\right], W\left(\mathbb{R}^{d}\right)\right)$ for some $T_{0}>0$ with $\left\|u^{\varepsilon}(t)\right\|_{W} \leqslant D$ for $t \in\left[0, T_{0}\right]$.

Moreover, from (1.3) and (2.2) it follows that $r^{\varepsilon}$ satisfies

$$
i \varepsilon \partial_{t} r^{\varepsilon}+\frac{\varepsilon^{2}}{2} \Delta r^{\varepsilon}=\varepsilon^{\alpha-\beta}\left(\mathcal{M}\left(u_{\mathrm{app}}^{\varepsilon}+\varepsilon^{\beta} r^{\varepsilon}\right)-\mathcal{M}\left(u_{\mathrm{app}}^{\varepsilon}\right)\right)-\varepsilon^{-\beta} R\left(u_{\mathrm{app}}^{\varepsilon}\right)
$$

with $\mathcal{M}(u)=\left(K *|u|^{2}\right) u$ for $t \leqslant \tau:=\min \left\{T_{0}, T\right\}$, and by Duhamel's formula and Lemma 3.2 iii. we obtain

$$
\begin{aligned}
\left\|r^{\varepsilon}(t)\right\|_{W} \leqslant & \varepsilon^{\alpha-\beta-1} \int_{0}^{t}\left\|\mathcal{M}\left(u_{\mathrm{app}}^{\varepsilon}(\tau)+\varepsilon^{\beta} r^{\varepsilon}(\tau)\right)-\mathcal{M}\left(u_{\mathrm{app}}^{\varepsilon}(\tau)\right)\right\|_{W} d \tau \\
& +\varepsilon^{-\beta-1} \int_{0}^{t}\left\|R\left(u_{\mathrm{app}}^{\varepsilon}(\tau)\right)\right\|_{W} d \tau,
\end{aligned}
$$

for all $t \leqslant \tau$. Writing

$$
\mathcal{M}(u+r)-\mathcal{M}(u)=\left(K *\left(u \bar{r}+\bar{u} r+|r|^{2}\right)\right)(u+r)+\left(K *|u|^{2}\right) r
$$


the estimate (3.3) gives

$$
\|\mathcal{M}(u+r)-\mathcal{M}(u)\|_{W} \leqslant\|\widehat{K}\|_{L^{\infty}}\left(3\|u\|_{W}^{2}+3\|u\|_{W}\|r\|_{W}+\|r\|_{W}^{2}\right)\|r\|_{W}
$$

Hence, replacing $u=u_{\text {app }}^{\varepsilon}$ and $r=\varepsilon^{\beta} r^{\varepsilon}$, and recalling $\beta>0$, we obtain for any $C>0$ and $\varepsilon_{0} \in(0,1]$, such that $3 \varepsilon_{0}^{\beta} C_{A} C+\varepsilon_{0}^{2 \beta} C^{2}=C_{A}^{2}$, that

$$
\left\|\mathcal{M}\left(u_{\mathrm{app}}^{\varepsilon}+\varepsilon^{\beta} r^{\varepsilon}\right)-\mathcal{M}\left(u_{\mathrm{app}}^{\varepsilon}\right)\right\|_{W} \leqslant \varepsilon^{\beta} C_{\mathcal{M}}\left\|r^{\varepsilon}\right\|_{W} \quad \forall \varepsilon \leqslant \varepsilon_{0}, \quad t \leqslant \tau
$$

where $C_{\mathcal{M}}:=4\|\widehat{K}\|_{L^{\infty}} C_{A}^{2}$, as long as $\left\|r^{\varepsilon}\right\|_{W} \leqslant C$.

Inserting the bounds (4.6) and (4.3) into (4.4), and recalling that $\varepsilon_{0} \leqslant 1, \alpha \geqslant 1$, $\tau \leqslant T$, we consequently obtain for $\beta \in(0, \gamma-1]$

$$
\left\|r^{\varepsilon}(t)\right\|_{W} \leqslant C_{R} T+C_{\mathcal{M}} \int_{0}^{t}\left\|r^{\varepsilon}(\tau)\right\|_{W} d \tau \quad \forall \varepsilon \leqslant \varepsilon_{0}, t \leqslant \tau .
$$

By Gronwall's lemma this yields

$$
\left\|r^{\varepsilon}(t)\right\|_{W} \leqslant C_{R} T e^{C_{\mathcal{M}} t} \quad \forall \varepsilon \leqslant \varepsilon_{0}, t \leqslant \tau .
$$

Hence, setting above $C:=C_{R} T e^{C_{\mathcal{M}} T}$ and $D:=C+C_{A}$ this estimate guarantees that the solution $u^{\varepsilon}$ exists on the whole time interval $[0, T]$, cf. the proof of Lemma 3.3. Moreover, recalling Lemma $3.2 \mathrm{i}$, we finally obtain the error estimate (1.5) which finishes the proof.

Acknowledgement. The authors want to thank Robert Schubert for pointing out to them the alternative approach described in Remark 2.2.

\section{REFERENCES}

1. T. Alazard and R. Carles, Semi-classical limit of Schrödinger-Poisson equations in space dimension $n \geq 3$. J. Diff. Equ. 233 (2007), no. 1, 24-275.

2. N. N. G. Berloff, Nonlocal nonlinear Schroedinger equations as models of superfluidity, J. Low Temp. Phys. 116 (1999) no. 5/6, 1-22.

3. R. Carles, Semi-classical analysis for nonlinear Schrödinger equations. World Scientific, Co. Pte. Ltd., Hackensack, NJ 2008.

4. R. Carles, WKB analysis for nonlinear Schrödinger equations with potential. Comm. Math. Phys. 269 (2007), no. 1, 195-221.

5. R. Carles, E. Dumas, and C. Sparber, Multiphase weakly nonlinear geometric optics for nonlinear Schrödinger equations, preprint arXiv:0902.2468.

6. R. Carles, N. Mauser, and H.P. Stimming, (Semi)classical limit of the Hartree equation with harmonic potential. SIAM J. Appl. Math. 66 (2005), no. 1, 29-56.

7. R. Carles and S. Masaki, Semi-Classical analysis for Hartree equation. Asymptot. Anal. 58 (2008), no. 4, 211-227.

8. M. Colin and D. Lannes, Short pulses approximations in dispersive media, SIAM J. Math. Anal., to appear. Preprint arXiv:0712.3940.

9. J. Giannoulis, A. Mielke, and C. Sparber, Interaction of modulated pulses in the nonlinear Schrödinger equation with periodic potential. J. Diff. Equ. 245 (2008), no. 4, 939-963.

10. H. Li and C.-K. Lin, Semi-Classical limit and well-posedness of nonlinear SchrödingerPoisson systems, Electronic J. Diff. Equ. 2003 (2003), no. 93, 1-17.

11. L. Hörmander, The analysis of linear partial differential operators I, Springer Verlag, Berlin 1983.

12. J.-L. Joly, G. Métivier, and J. Rauch, Coherent nonlinear waves and the Wiener algebra, Ann. Inst. Fourier 44 (1994), no. 1, 167-196.

13. P. Kirrmann, G. Schneider, and A. Mielke, The validity of modulation equations for extended systems with cubic nonlinearities, Proc. Roy. Soc. Edinburgh Sect. A, 122 (1992), 85-91,

14. P.-L. Lions and T. Paul, Sur les mesures de Wigner, Rev. Mat. Iberoamericana 9 (1993), no. 3, 553-618. 
15. H. Liu and E. Tadmor, Semi-Classical limit of the nonlinear Schrödinger-Poisson equation with subcritical initial data, Methods Appl. Anal. 9 (2002), no. 4, 470-484.

16. P. A. Markowich and N. J. Mauser, The classical limit of a self-consistent quantum-Vlasov equation in 3D, Math. Models Methods Appl. Sci. 3 (1993), no. 1, 109-124.

17. C. Sulem and P. L. Sulem, The nonlinear Schrödinger equation. Springer Series on Applied Math. Sciences 139, Springer (1999).

18. P. Zhang, Wigner measure and the semi-classical limit of Schrödinger-Poisson equation, SIAM J. Math. Anal. 34 (2002), no. 3, 700-718.

19. P. Zhang, Y. Zheng, and N. J. Mauser, The limit from the Schrödinger-Poisson to the VlasovPoisson equations with general data in one dimension, Comm. Pure Appl. Math. 55 (2002), no. $5,582-632$.

(J. Giannoulis) Zentrum Mathematik, Technische Universität München, BoltzmannStrasse 3, D-85747 Garching B. München, Germany

E-mail address: giannoulis@ma.tum.de

(A. Mielke) Weierstrass-Institut für Angewandte Analysis und Stochastik, MohrenStrasse 39, 10117 Berlin and Institut für Mathematik, Humboldt-Universität zu Berlin, Rudower Chaussee 25, 12489 Berlin, Germany

E-mail address: mielke@wias-berlin.de

(C. Sparber) Department of Applied Mathematics and Theoretical Physics, CMS, Wilberforce Road, Cambridge CB3 0WA, England

E-mail address: c.sparber@damtp.cam.ac.uk 